\title{
Review of: "Growth, yield and quality of two guar (Cyamopsis tetragonoloba L.) ecotypes affected by sowing date and planting density in a semi-arid area"
}

weeradej meeinkuirt ${ }^{1}$

1 Mahidol University

Potential competing interests: The author(s) declared that no potential competing interests exist.

Many crop plants require water, but many of them grow in arid areas with limited water supplies, making this finding intriguing in terms of potential applications. Thus, choosing crop plants that can grow in arid or semi-arid environments while still producing high-quality harvests is critical. The authors experimented with growing two guar ecotypes in Iran, which served as study plants and economic crop plants. Overall, yield production of the study plant is influenced by ecotype, plant habit, and agro-climatic environment. The following are some of my questions and comments:

1. I'm not sure where you got your rainfall and temperature data. Is the information averaged?

2. I find the order in which the authors write in the paper to be really strange. For example, the authors presented soil texture in the field site after adding rainfall and temperature data. Those sentences, in my opinion, were not connected. The physicochemical properties of the soils in Table 1 should be the information in the statement (Just suggesting)

3. I'm curious about the differences in plant density between the plots.

4. 3) I'm curious as to why the authors utilized urea as a nitrogen fertilizer ( $\left.150 \mathrm{~kg} \mathrm{~N} \mathrm{ha}^{-1}\right)$ in the form of Urea $(46 \% \mathrm{~N})$. Is that the rate in the study site for traditional crops?

5. Table 1's data should be arranged more properly.

6. The authors presented physicochemical properties of soils, however they did not mention it specifically or discuss it in depth. What is a key factor that affects plant development and yields?

7. Authors should include a critical physicochemical property of soils that affect plant growth and yields in their conclusions.

8. The journal's first letter in bibliography should be capitalized. For example, Crop Science. Please be cautious and read the journal's author instructions. 